\title{
Thinking in action
}

\author{
Stephen Cowley · Frédéric Vallée-Tourangeau
}

Published online: 2 February 2010

(c) Springer-Verlag London Limited 2010

\section{People in co-acting assemblages}

While computers can be used to model human competencies, formalization has its limits. Sensori-motor dynamics are probably necessary to intelligence. Applied to language, verbal patterns become constraints or, in Elman's (2004) terms, cues to meaning. Unlike symbol processors, humans act, mean and use the feeling of thinking (Harnad 2005). While language has an artificial (or formal) aspect, human intelligence is embodied. In spite of widespread belief to the contrary, brains do not need to generate sets of sentences. In challenging code views of language, we find parallels with the complex systems we call cells. Given DNA code-makers, formal features constrain protein synthesis. Life, Barbieri (2007) argues, can be traced to natural artifacts. ${ }^{1}$ This parallels how culture enables us to bring biodynamics under the control of physical and non-physical (or cultural) patterns. In turning from physical symbol systems, weight falls on DEEDS: human thinking is Dynamical, Embodied, Embedded, Distributed and Situated (Walmsley 2008). Our actions are situated in a normative world where

Guest editors: Stephen Cowley and Frédéric Vallée-Tourangeau.

S. Cowley $(\square)$

School of Psychology, University of Hertfordshire,

College Lane, Hatfield, Hertfordshire AL10 9AB, UK

e-mail: s.j.cowley@herts.ac.uk

S. Cowley

University of Kwa-Zulu Natal,

Durban, South Africa

F. Vallée-Tourangeau

Psychology Research Unit, Kingston University,

Kingston-upon-Thames, Surrey KT1 2EE, UK

e-mail: f.vallee-tourangeau@kingston.ac.uk bodies use learning (and other interactional products) in helping us to co-ordinate internal and external structure. As explained in the following paragraphs, people manage what are termed 'co-acting assemblages'. Using cognitively inspired ethnography, Hutchins (1995) saw the importance of close coupling in how a crew used artifacts to bring a ship into port. In his terms, sailors, procedures and instruments constitute distributed cognitive systems. What happens 'in the head' is inseparable from actions, speech and how instruments serve to track the world. While what we do makes extensive use of documents, speech and sign, cognition is grounded in bodily co-ordination. Cultural history shapes our doings, customs, means of production, and social institutions. Thinking links the natural artefacts of DNA with brains that give us skills in using the artefacts and norms of our communities.

Human cognition, like problem solving, is cultural. While emphasized in, above all, Vygotsky and Wittgenstein, the idea has become more prominent in cognitive science. In seeking a way between Vygotsky's mentalism and Wittgenstein's conceptualism, humans can be seen as ecologically special (Ross 2007). Drawing on biology and strategic co-ordination, human cognition uses historically derived procedures (including language), artifacts and modes of organization (e.g., naval academies). Distributed cognition (DCog) allows people to connect culturally specific artifacts, technologies and institutions: human sense-making occurs within meshworks that include the

\footnotetext{
${ }^{1}$ Genes and proteins are assembled by molecular robots on the basis of outside instructions. For Barbieri, as manufactured molecules, they differ from ordinary molecules as do artificial and natural objects. Thus, if molecules are "natural when their structure is determined from within, and artificial when it is determined from without, then genes and proteins can truly be referred to as artificial molecules, as artifacts made by molecular machines (2007, p. 183)."
} 
verbal patterns of languages. In Clark's (2003) phrase, we are natural born cyborgs. While he stresses technology, older traditions give similar insights. Not only is the cultural central to Vygotsky but, by intellectual tradition, it also dominates Marx and Hegel. In broad terms, these approaches recognize, first, it is by and through activity that human subjects create language. Second, in the course of evolution, language gave rise to the division of labour, social practices and history. Not only can we think silently but, at the same time, we can draw on distributed cognitive systems.

\section{From distributed language to thinking}

The Special Issue brings these traditions into contact while, at the same time, focusing on how cultural patterns constrain cognitive dynamics. By focusing on thinking in action, we treat Baber et al.'s (2006) 'resources for action' as central to cognition. This approach was catalyzed by a 2008 Symposium at the University of Hertfordshire organized by the Distributed Language Group. This group advocates a reconceptualization of language as first-order activity which, remarkably, is heard against second-order cultural constraints (verbal patterns). It is the hybridity of language that allows infants to use co-ordination in learning to engage with (and contribute to) the language meshwork of their community. Indeed, the rewards of social activity prompt them to become living subjects or persons. Building on this view, the Hertfordshire symposium focused on distributed thinking. Action and interaction thus became the domain of analysis that focuses on "co-acting assemblages." By introducing this term, participants were asked to consider what "humans do with what they know (knowledge) and how their intelligence plays out." Of the papers of the Special Issue, five emerged from the resulting interaction (Perry, Spiekermann, Baber, Vallée-Tourangeau \& Wrightman, Jones). The Editors added others to show, first, the power of thinking with external representations (Kirsh) and, second, how second-order constraints affect thinking (Ben Naim et al.). In co-acting assemblages, thinking is simultaneously controlled on the scales of brains, interactions and changing cultural resources. Given this multi-scalar view, the symposium participants examined cognitive processes involved in looking over a desk drawer, making words in Scrabble, and in the slower and faster scales of designing roads and deciding to launch nuclear missiles. To integrate the relevant events, an agent or agents depend on action (or interaction). Decisions thus emerge in the cognitive dynamics of brain, body and world. Indeed, it is because of interest in slow processes like crime scene investigation and winning trust that we broaden our focus from distributed cognitive systems to co-acting assemblages. By asking how these function, we avoid the assumption that they depend on a stable biological core (the brain). In extending the ecological view, we regard cognitive dynamics as linking events within the skin with artifacts, patterns and modes of action that call up our and other people's experiences. Brains ready us for shifting between ways of acting that lead to the configuration and dissolution of assemblages. Human ways of acting (and attending) presuppose a reciprocal relation with environmental conditions. Accordingly, we see our work as extending distributed cognition.

\section{Cognition in the wild}

Since Hutchins (1995) published Cognition in the wild, distributed cognition has spread slowly. In the Cambridge handbook of situated cognition, the 'primer' focuses on how cognition is embodied, embedded and extended (Robbins and Aydede 2008). By contrast, given ethnographic origins, the distributed view stresses persons-embedded-in-actionand/or-interaction. Emphasis on assemblages provides a subtler challenge to internalism than does appeal to embodied, embedded and/or extended minds. Distributed cognition has thus excited less philosophical interest. Nonetheless, it has many important applications and, from a theoretical perspective, is crucial to rethinking the nature of language. ${ }^{2}$ For, if language cannot be localized, it becomes "a contextually determined process of investing behaviour or the products of behaviour (vocal, gestural or other) with semiotic significance" (Love 2004, p. 530). Leaving semiosis in the background, the Special Issue focuses on thinking. In the terms of DCog tradition (see Hollan et al. 2000), this can be characterized as follows: (1) Cognitive processes are distributed across the members of the social group; (2) cognitive processes involve co-ordination between internal and external structure; and (3) processes are distributed through time in such a way that the products of earlier events transform the nature of later events.

These theses apply to all the work described in the following paragraphs. Accordingly, we begin with the practical and theoretical importance of such views. Drawing on ethnography, Perry uses the model in consultancy for the construction industry. In this context, he shows how people move in and out of assemblages as they undertake tasks that are irreducible to procedures. In managing road construction even the absence of shoes (or mud on boots!) can be used as resources for action. Next, Kirsh shows the

\footnotetext{
${ }^{2}$ DCog serves in fields that include education (Cox 1999), learning to use brain images (Alač 2005), science studies (Giere and Moffat 2003) and medicine (Patel et al. 2004).
} 
fundamental importance of such assemblages for cognitive theory. Access to external representations enable humans to develop routines of project and materialize. Rather than invoke a hypothetical problem space, both papers explore how skills with assemblages enable decisions to exploit what Kauffman (2000) calls the adjacent possible. Our actions and interactions are thus managed in living spaces that are conditioned by culture as well as biology. How is this achieved? Pursuing this, the next papers formalize contextual functions that arise from an individual history of using social norms. Like Spiekermann, Ben Naim and colleagues investigate what we experience as social statics-modes of organization that constrain (inter)actions. Whereas Ben Naim et al. focus on web-mediated trust, Spiekermann turns to judgement aggregation. Formalization is thus of value in naturalizing social functions. Just as for language, much depends on virtual patterns (secondorder cultural constructs) that constrain what people do as they (inter)act. Next, we downshift the time-scale. We ask how interaction can be investigated with respect to parts and modes of organization. First, Baber sketches how and why crime scene examiners act and, given legal concerns, he offers a conservative definition of cognition. While assemblages use objects, he emphasizes narratives-and how these are construed. In a legal setting (among others), these bring bias into play. After that, we turn to Scrabble. Using experimental methods, Vallée-Tourangeau and Wrightman show that solutions use trade-offs between neural function and real-time action/interaction. While brains matter, we deal with a complex Scrabble problem by drawing on experience of a systemic world. Finally, Jones uses integrational theory to show how far we still have to go. While applauding the turn to culture, he complains that such approaches neglect the human subject. Appeal to assemblages obscures the moral texture of human lives.

\section{What next?}

We identify resonances and dissonances relevant to naturalizing human cognition and, specifically, distributed thinking. Using specific circumstances, people becomenot (pure) information processors-but active users of Baber et al.'s (2006) resources for action. Stretching DCog, we then ask how social context constrains thinking and, conversely, how co-acting assemblages affect culture's slow dynamics (norms, procedures and modes of organization that individuals perceive as social 'statics'). Computing systems are shown to be of value in 'cognitivising' trust and as group decisions sensitize to modes of organization (e.g., dictatorship). Next, we ask what is truly cognitive if, as here, action and interaction are fundamental. Surprisingly, having used DCog to develop technologies for crime-scene investigation, Baber returns to mental states. Countering, Vallée-Tourangeau and Wrightman show that-far from being just mind stuffcognition links internal and external resources. Finally, Jones reminds us that, in spite of its systemic nature, this view fails to consider power, passion and politics or how we tell right from wrong.

\section{The papers recontextualized}

Recognizing that we use 'resources for action' has important practical and theoretical outcomes. In DCog ethnographic tradition, Perry turns from the routine-based procedures of armies and navies (cf. Hutchins 1995). Instead, he focuses on loosely coupled systems used in making decisions about road construction. Brain-bound intelligence, he shows, uses "technical, social and organizational systems" (Perry, this issue). Far from being controlled by individuals, events also depend on procedures, resource flexibility and how people see what they should do. Challenging task analysis, Perry shows how situation awareness contributes to events that happen on the fly: since we track each other's doings, office design can save a construction company time and money. The focus falls on thinking 'in action'. Given ethnographic methods, we shift from task analysis to scales that give meaning to action. Thus, ethnography is itself a resource that constrains our understanding. On another tack, Kirsh uses classic DCog to focus on processes that can contribute to silent thinking. He chooses to work with the language of representation, process, transformation, co-ordination. In these terms, he opens up a big question: Given on-board intelligence, why do we create external representations? He suggests that human intelligence permits us to construct quasi-symbols. Sign-making resources enable us to project and materialize. While goals and inner representations are not excluded, results that materialize allow us to process information accurately, deeply and precisely. Typically, we construct a static entity or a "persistent element that can be measured or identified and reliably identified and re-identified" (Kirsh, this issue). Thus, material (measurable) inscriptions or patterns serve as quasi-symbols. Given experience, we evoke referents by projecting what results from interaction. When stabilized in a drawing (or transcription), they can facilitate future judgements. Quasi-symbolic patterns may, for example, link a visuospatial store with working memory. Using external representations, brains prompt us to bring off positive results. However, the language of $\operatorname{cog}$ nitive science keeps Kirsh focused on co-ordination, the glue of cognition (Kirsh 2006). The work he presents here does not explore how actions gain coherency as we concert our bodies. Addressing how circumstances which make 
this possible, the next papers address the cultural framing of cognition.

Human cognition occurs in action and interaction. Since our modes of acting unite the scales of history with biodynamics, it cannot be reduced to real-time events. In dialogue too, first-order languaging co-occurs with slow patterns or 'words'. Pursuing events in these cultural scales, Ben Naim and his colleagues turn to practical matters and money-making. Tongue in cheek, they formalize 'trust' by stripping this down to a cognitive component (leaving emotion and responsibility aside). In principle this could be implemented by web-based systems and, if it works, this has theoretical, practical and financial implications. Calling this 'distributed information processing', they focus on results. Stable norms can influence subsequent thinking. Interactions are aggregated in ways that constrain future actions-where an agent has the capacity to use slow-scale systems. This has implications for both Jones's critique and biological systems that can draw on each other's experience. In similar ways, it permits distributed cognition to come into dialogue with both Social Choice theory and Social Epistemology. Spiekermann addresses collective thinking that formalizes group decision making. This applies where detectives decide on an arrest, juries whether to convict and governments on resolving a banking crisis. They overcome the discursive dilemma-many decisions cannot be resolved procedurally. Nonetheless, truth-tracking and coherence can be formalized. Spiekermann builds on Condorcet's thesis that, at times, groups make good decisions using only 'better than random' information. Highlighting aggregating judgements and the qualities of truth-tracking, these models have political importance. Thus, while Ben Naim and colleagues formalize how 'trust' influences decision-making, Spiekermann tracks the analytical and normative dimension of flexible, adaptive behaviour. The context of thinking shapes action/interaction: as a result, modes of organization exert trickle down effects on our lives. While, a reader must decide if this complements or challenges distributed cognition, new questions open up. If thinking is dynamic, involves body-world interaction and gives rise to higher level statics, what we do and say is affected by analytical and normative system-resources (quasi-symbols and other structures). Modes of organization constrain the actions and thoughts of groups, dyads and individuals. Indeed, using formalist tradition, Spiekermann argues that the approach has implications for decision-making and also aspects of our lives affected by public policy, our preferences, and access to resources and emotions.

Having touched on social context, the next papers focus on how assemblages induce us to think. Turning from ethnography and formalization, they make no appeal to representation. Baber focuses on crime scene investigation from the perspective of a technological innovator. Stepping back from Extended Mind, he focuses on how things are and should be done. Like Harnad, he invokes mental states: "It is not 'cognition' that is distributed so much as objects-in-the-world which play a part in supporting, structuring and aiding the activities of cognition" (Baber, this issue). As a result while said to be trivially distributed between people, cognition is also mediated "through the collection, manipulation and dissemination of artifacts" (this issue). In using objects as affordances, cognition is 'weakly' distributed and, in accomplishing tasks (alone or in groups), we find a 'strong' form of distributed thinking. Turning from 'loosely coupled systems', Baber emphasizes language or, rather, narrative. This is crucial in that investigators rely on developing story about a criminal's actions. Second, crime scene examiners use both formulations and informal language. Not only do they rely on minimal verbal descriptions (often aided by technological and photographic means) but they also talk with colleagues and draw on verbally framed inferences. In the crime scene, narrative sets up biases that can be good or bad. Linking these with 'situational awareness' Baber uses DCog to train others in (a) using affordances and (b) managing distributed tasks. He thus refocuses on what subjects actually do. By contrast, Vallée-Tourangeau and Wrightman prefer experimental rigour. Espousing a systemic view, they treat cognition as "configured by interactive links between internal and external resources" (this issue). Avoiding appeal to an unchanging organic system (the brain), they link observations of how people generate words with Scrabble tiles with measures of individual differences in verbal fluency. Instead of asking about putative competencies, they stress interactions between neural and non-neural resources. They find a delicate interplay between objects, task difficulty and a subject's verbal fluency. This opens a way to a dialectics of experimentation focused on the actual use of external resources. By implication social statics function rather like cognitive habits. These too provide a "web of reciprocal influences among elements that configure a functional context in which the agent is embedded" (Vallée-Tourangeau \& Wrightman, this issue). Thinking-in-an-fMRI scanner is thus unlikely to resemble thinking in action. Rather than ask what resources are used (or how they influence us), task difficulty induces individuals to link their brains, hands and objects. In Kirsh's words, "cognitive processes migrate to wherever they are best or mostly easily performed" (this issue). Generally, systems result from interactions. Alongside methodological innovation, the approach shows the gain in applying standard methods to study co-acting assemblages. 
Recognizing that cognition depends on more than a naked brain has many practical and theoretical consequences. Jones, however, disturbs any smugness. While an improvement on reifying people as information processors, Jones thinks we need to more than ask what cognition is for and how it works. With the help of Hollywood glamour, he stresses that assemblages have little to do with morally responsible human beings. In his view, such talk is little better than appeal to inner programs. We cannot describe decision-making independently of rights and wrongs. Premeditated acts, Jones claims, always have a moral dimension. Using Crimson Tide, Jones focuses on how the fictional hero of a film challenges procedure-driven action. Since human life draws on what Hutchins (1995), perhaps glibly, calls a 'cognitive, cultural process', we lose sight of what things mean for people. In Love's (2004) terms, individuals also "attach signification to signs". Without an account of how this is done, little progress will be made with thinking in action. Human agency has an irreducible moral texture. Having abandoned inner mind, we need to clarify what is human. For Jones, this depends on how living beings draw on total situations to attach meaning to the doings of others.

\section{Distributed thinking and results}

If thinking occurs during interaction, as we draw on both biodynamics and social constraints, we need to focus on the results of co-ordinating. These, moreover, will be associated more closely with groups than individuals. Indeed, if Condorcet is right, thinking as groups may be crucial in developing human understanding. How, then, do thoughts resonate across this set of papers? Strikingly, all converge on one, rather unexpected, point: Human cognition is systemic.

Far from arising in a single brain, human action and interaction also draw on social meshworks. Cognition is neither modular nor based on general processing but, rather, integrates resources across time-scales. Given its systemic nature, interactions link statics (e.g., procedures and norms) with modes of action-and informal narratives - that arise as we make sense of our doings. This parallels the distributed view of language where secondorder cultural constructs (especially words) constrain interactional events that result from pico-scale expression. In terms of social structure, we may conform to cognitive trust and thereby allow groups to influence our judgements. While compatible with Hollan et al.'s (2000) view of distributed cognition, the focus shifts to results. "Processes are distributed through time in such a way that the products of earlier events transform the nature of later events" ( $\mathrm{p}$. 176 emphasis added). What consequences arise from shifting emphasis from co-acting assemblages to how manipulations affect results?

While the papers concur on much and offer a myriad of disagreements, we are struck by two dissonant voices. The first raises the question: Is cognition to be identified with mental states? While the second asks: How are we going to account for the living subject? In internalist work, cognition is often modelled by functions that use, say, symbol processing or neural networks. What the Special Issue shows is that formalization also illuminates aspects of cognition that operate beyond the body (such as in using the internet). Thus, just as language can be formalized as a set of sentences, 'trust' can be reconceptualized to fit machine mediated application. For some purposes, this is adequate: For example, Bechtel et al. (1998) argue cognitive science moves forward by focusing on-not the nature of cognition-but what it is for (in an evolutionary context) and how it works. Most of the authors (but not Jones) would share this view. Yet, like Harnad (2005), Baber takes the conservative view that cognition identifies mental states lying between input-output systems. To an extent at least, Jones would agree: by underplaying the individual, he thinks, we lose sight of our moral nature. Accordingly, he proposes a contra-disciplinary view where moral responsibility is deemed central to human lives. It would be interesting to encounter a risposte to this view. There is, however, a way of using Harnad's challenge to develop an alternative to input-output models. Likening cognition to a headache, Harnad (2005) suggests that if it is not the property of one individual, several persons must all have headaches. The analogy with a headache thus places the feeling of thinking 'in' the head: for Harnad, it occurs between input and output. By contrast, Thompson (2007) or Järvilehto (2009) would argue that the feeling of thinking is intrinsic to action/interaction. Memory, motivation and anticipation all use kinaesthetic and enkinaesthetic senses. ${ }^{3}$ In meeting Jones's challenge, therefore, we might ask how moral texture co-emerges with capacities from acting in social roles. This can be traced, in large part, to the neural (and mental) events that co-emerge with cognitive dynamics of acting, feeling and thinking. While people indeed 'attach significance to signs', Jones would surely agree, that this can only occur within the adjacent possible.

Placing cognition in the context of thinking in action brings out its systemic nature. DCog can thus be more clearly placed in historical context. This shows, as Jones makes clear, that there is a clear break with Vygotsky and

\footnotetext{
3 The term refers to a reciprocal affective neuro-muscular dynamical flow and muscle tension felt between an agent and the world (or other agents): anticipatory dynamics can be experienced through both direct and indirect touch, being physically touched and perception of others perceiving you (Stuart 2009).
} 
activity theory tradition. Given its historical roots, individuals came to be seen, in our terms, as contributing to the assemblages that function within a cognitive ecology. While based in Hutchins's cognitive ethnography, the approach can be developed in many ways. Thus, Kirsh, for example, relies on the language of Cognitive Science, and Baber uses crime scenes to develop his grasp of DCog. Nonetheless, representation, transformation, process and co-ordination all derive from thinking about models. However, even where the nature of the system remains unspecified, such terms separate the cognizer from the world. In Järvilehto's (2009) terms, they mistakenly separate the brain and/or body from what it can do. Systemic psychology, by contrast, begins with the anticipatory powers of biology that enable an organism to become a person.

"The agent of psychological processes is not the brain or body but a person who is a dynamical product of social relations and common results. Psychological concepts refer to the different aspects of the organization of the continuous system and cannot be located in any local parts, such as parts of the brain" (Järvilehto 2009: 117).

While striking a chord with Kirsh's recognition that 'quasi-symbols' result as we project and materialize, this terminology omits what they mean for the person. ${ }^{4}$ In tracing diagrams (and texts), we use physical surrogates of virtual patterns. ${ }^{5}$ Social structure anchors a history of sensing-as-we-act. The time scale of Kirsh's analysis of the mind-body-world interaction is different from the slow dynamics that use virtual patterns such those of Ben Naim et al.'s 'cognitive trust' or Spiekermann's 'modes of operation'. DCog also self-organizes in the slow timescales of history.

The papers all focus on results. These serve Perry in selecting aspects of the world that inform valuable decisions about road building. They allow Kirsh to examine how quasi-symbols give rise to (what may be) humanspecific forms of thinking-ones based on "shareable and identifiable objects of thought" (this issue). In relation to social context, interactions can result in virtual constraints that shape, for example, trust and good decisions. Spiekermann's discursive dilemma shows that much of what we

\footnotetext{
${ }^{4}$ While recognizing that the person is not reducible to a biological organism, Järvilehto takes no stance on whether, as argued here, persons rely on skills in configuring and dissolving assemblages.

${ }^{5}$ For some, this is central to the distributed view of language: as we monitor both our own speech and that of others, we hear and react to virtual constructs-verbal patterns that we have learned to picture as akin to written words (see, Cowley 2007; Love 2007; Ross 2007). Regarding these as real leads to the written language bias which, for Linell, led twentieth century linguistics down a blind alley.
}

do-and value-is irreducible to procedures. We sense what is appropriate and, in so doing, act on the fly. At these cracks, Spiekermann finds politics and Baber offers views on how the law should-and should not-proceed. Perhaps, subjects emerge as we act to configure and dissolve the assemblages that fall under our control. Appeal to modes of organization, procedures and actions/interactions is, moreover, consistent with how science views mechanism (viz. systems exploit parts, procedures and modes of organization, see, Bechtel 2008). Further, this supports the view that grammatical and lexical aspects of language are second order patterns. Like 'dictatorship' or 'trust', they need be neither algorithm-like nor physically realized. Rather as modes of organization, they draw on a history of co-ordination. Having recognized the power of narrative in earlier work (Baber et al. 2006), Baber now stresses how it biases us. Like social statics, narrative adds a spin to events. In Vallée-Tourangeau and Wrightman's work, habit-based equivalents are captured by measures of verbal fluency. A history of languaging thus has a measurable impact on word production tasks. Unknowingly, task difficulty prompts us to switch cognitive strategies (see also Kirsh 2008). Broadly, the harder the task the more external resources can offer. This is not inconsistent with Jones's view that, to understand moral decisions, one must look at the values associated with what we do-and, presumably, in understanding living subjects-to consider how these result form interactional history (including experience of language).

\section{Conclusion}

Järvilehto (2009) proposes that psychology be founded on investigating the results of actions. Viewing humans as organism-environment systems, he rejects computational models (not methods) to stress that as organisms anticipate they also act. What happens depends on using circumstances in ways that are flexible and adaptive. Far from being intrinsically goal directed, cognition is action/interaction based on familiarity with a systemic world. In this way, the strengths of ecological psychology link up with mainstream cognitive science. In what domain can this be pursued? Strikingly, all the papers of the Special Issue focus on loose coupling with the world. Using results, we develop skills that, in a broad sense, contribute to solving problems. Thus, the next Distributed Thinking Symposium will focus on rethinking problem solving. While the concept of a functioning system is valuable, we also need to address how systems exploit action and interaction as they connect across time-scales. Indeed, assemblages are configured and dissolved as we escape from procedures by acting (e.g., using the feeling of thinking) in a social world 
(e.g., trust). Methodologically, a focus on systemic thinking may clarify how communities tackle general issues. To understand thinking, we can ask how the meshwork constrains people as they integrate internal and external resources. It thus matters that cognitive dynamics show sensitivity to social statics_-slower dynamics_-based in a group's history. And, given dysfluencies-systemic collisions - cognition can draw on the feeling of what happens. This is analogous to Barbieri's (2007) view of how cells use statics (DNA sequences) to manufacture proteins. This systemic view identifies an executive (an RNA system) that links embodied processes with arbitrary sequences (DNA) to synthesize context-relevant proteins. Thus, as cells selfmanufacture, assemblages use us to self-configure. Social demands induce us to anticipate rewards that may be available in (perceived) circumstances. Counterbalancing the current fashion for embodiment, this approach traces interaction, language and society-not to neural processes-but brain-body-world encounters.

\section{References}

Alač M (2005) From trash to treasure: learning about brain images through multimodality. Semiotica 156:177-202

Baber C, Smith P, Cross J, Hunter J, McMaster R (2006) Crime scene investigation as distributed cognition. Pragmat Cognit 14:357385

Barbieri M (2007) Is the cell a semiotic system? In: Barbieri M (ed) Introduction to biosemiotics: the new biological synthesis. Springer, Dordrecht, pp 179-208

Bechtel W (2008) Mental mechanisms: philosophical perspectives on cognitive neuroscience. Routledge, London

Bechtel W, Abrahamsen A, Graham G (1998) The life of cognitive science. In: Bechtel $\mathrm{W}$, Graham $\mathrm{G}$ (eds) A companion to cognitive science. Blackwell, Oxford, pp 1-104
Clark A (2003) Natural-born cyborgs: minds, technologies and the future of human intelligence. Oxford University Press, New York

Cowley SJ (2007) Cognitive dynamics and distributed language. Lang Sci 29:575-583

Cox R (1999) Representation construction, externalised cognition and individual differences. Learn Instruct 9:343-363

Elman JL (2004) An alternative view of the mental lexicon. Trends Cognit Sci 8:301-308

Giere RN, Moffatt B (2003) Distributed cognition: where the cognitive and social merge. Social Stud Sci 33:301-310

Harnad S (2005) Distributed processes, distributed cognizers and collaborative cognition. Pragmat Cognit 13:501-514

Hollan J, Hutchins E, Kirsh D (2000) Distributed cognition: toward a new foundation for human-computer interaction research. ACM Trans Comput Hum Interact 7:174-196

Hutchins E (1995) Cognition in the wild. MIT, Cambridge

Järvilehto T (2009) The theory of the organism-environment system as a basis of experimental work in psychology. Ecol Psychol $21: 112-120$

Kauffman S (2000) Investigations. Oxford University Press, Oxford

Kirsh D (2006) Distributed cognition: a merthodological note. Pragmat Cognit 14:249-262

Kirsh D (2008) Problem solving and situated cognition. In: Robbins R, Aydede M (eds) Cambridge handbook of situated cognition. Cambridge University Press, Cambridge, pp 264-306

Love N (2004) Cognition and the language myth. Lang Sci 26:525544

Love N (2007) Is language a digital code? Lang Sci 29:690-709

Patel VL, Arocha JF, Zhang J (2004) Thinking and reasoning in medicine. In: Holyoak K, Morrison RG (eds) The Cambridge handbook of thinking and reasoning. Cambridge University Press, Cambridge, pp 727-750

Robbins P, Aydede M (2008) A short primer on situated cognition. In: Robbins P, Aydede M (eds) The Cambridge handbook of situated cognition. Cambridge University Press, Cambridge, $p p$ $3-10$

Ross D (2007) H. sapiens as ecologically special: what does language contribute? Lang Sci 29:710-731

Stuart S (2009) The mindsized mashup: mind isn't supersized after all. Analysis. doi:10.1093/analys/anp122

Walmsley J (2008) Methodological situatedness; or, DEEDS worth doing and pursuing. Cognit Syst Res 9:150-159 